\title{
Pulsed Eddy Current Imaging of Inclined Surface Cracks
}

\author{
Faris Nafiah, Ali Sophian \\ International Islamic University Malaysia \\ Jalan Gombak, 53100, Kuala Lumpur, Malaysia \\ e-mail: ali_sophian@iium.edu.my
}

\begin{abstract}
Inclined fatigue cracks can potentially cause severe damage to metallic structures as they affect larger region in the tested structure compared to crack perpendicular to the sample surface. The abilitiy to detect and characterize such cracks is paramount in non-destructive testing (NDT). Pulsed eddy current testing (PEC) is known to offer a broadband of excitation frequencies, which in conjuction with $C$-scan imaging, may offer discrimination of inclination angles of cracks. Finite element modelling (FEM) was carried out to study the effects of different crack inclination angles, while experimental results were used to verify the FEM results. Selection of both time and frequency domain features for $C$-scan image construction was also presented, where C-scan images of peak value and amplitude at $200 \mathrm{~Hz}$ were shown to be potentially capable in determining different inclination angles. Nevertheless, between these two signal teatures, the amplitude at $200 \mathrm{~Hz}$ was found to be more effective in the discriminataion of inclined cracks.
\end{abstract}

Keywords: non-destructive testing, pulsed eddy current testing, inclined cracks, temporal and spectral analysis

\section{Introduction}

Belonging to the electromagnetic non-destructive testing (NDT) category, pulsed eddy current testing (PEC) is renowned for its beneficial wideband excitation of magnetic field into a tested metallic structure [1]. This induces transient eddy currents into the structure, which in turn, induces a secondary magnetic field opposing the primary magnetic field. A sensing element senses the resultant magnetic flux density, thereby giving the information on the flaw or defect due to the change in the structure's material property [2]. Three typical signals are present in the general application of PEC, namely reference, response and differential signals. Reference signal refers to a resultant magnetic field picked up by the sensing element when no flaws are present. Response signal is the resultant magnetic field when the induced eddy current is perturbed by the presence of flaws while differential signal is the subtraction of reference signal from the response signal [3]. Hence, a differential signal basically contains the information due to the change in the induced eddy currents.

Eddy current's penetration is governed by the skin effect where at the standard skin depth its density is reduced to $1 / \mathrm{e}$ of the original density at the surface. The equation for the skin depth is as shown below [3]:

$$
\partial=\frac{1}{\sqrt{2 \pi f \mu \sigma}}
$$

, where $\partial$ is skin depth, $f$ is frequency of the current, $\sigma$ are $\mu$ are electrical conductivity and magnetic permeability of the specimen respectively. As a pulse waveform consists of a broadband of frequency components, the induced eddy current that is present in a short period contains information on a wide range of depths. The most used features extracted from differential signal in time domain is peak value and peak arrival time [3]. Peak value describes how severe the decline of induced eddy current due to the crack [4]. Meanwhile, the time at which the peak value occurs describe the depth information, in which the early time data corresponds to the information on shallow parts of the sample, and the subsequent later time explains otherwise [5]. As much as resourceful temporal analysis is, PEC signals can also be 
evaluated in spectral analysis. Equation (1) shows that high frequency components are more sensitive to the region near to the surface of the sample while low frequencies would be more sensitive to flaws buried deeper into the sample [6].

In PEC, imaging techniques are devised by constructing a selected feature from the differential signal according to its spatial position. The spatial mapping of selected features makes up a planar view of the tested structure, which is commonly referred as a C-scan image. $\mathrm{C}$-scan images provide a more intuitive interpretation on the crack structure. C-scan imaging can be achived by scanning the probe throughout the tested sample [7]-[10],or by using sensor arrays [9]. Using scanning, He et. al proposed the use of rectangular excitation coil to provide a more evenly distributed magnetic field into the sample to effectively quantify the crack widths and lengths [7]. In another work, a probe with a ferrite core placed between the excitation and pickup coil was proposed to allow enhancement of the differential signal, thus impoving the Cscan images [8]. Besides scanning, a handheld technique has also been proposed, where a driver trace was placed between the $4 \times 4$ arrays of pickup coils. The driver traces were excited separately, hence whenever a crack is present underneath one of the pickup coils, a defect signal will be sensed [9].

A long list of works on defect analysis using PEC have been reported, ranging from defect classification to defect quantification. However, research on using PEC on inclined crack has not been found by the author, except a work regarding the use of Fisher's LDA for crack classification [11]. Neglecting angle of crack tends to lead to crack size underestimation, as inclined crack bears a larger affected area than a crack perpendicular to the specimen surface. Many research gravitates towards using other NDT techniques on analysis of inclined cracks, such as PEC thermography (PECT), ultrasound testing (UT), and electromagnetic acoustiv transducer (EMAT). PECT was employed as varying surface crack angles clearly gives different maximum temperatures observed through the thermal camera [12]. Other than that, Rosli et al. claimed that the approximation of slot inclination using EMAT can be done using calculation of ratio of the enhancement factors of the in-plane to out-of-plane Rayleigh wave velocity components, or also termed as FER [13].

This work opens up the utilization of PEC on analysis of inclined cracks. The effects of magnetic field at different spatial positions are investigated by using both finite element modelling (FEM) and experimental analysis. Features extracted from time and frequency domain are analysed to construct $\mathrm{C}$-scan images to provide an understanding on the effects of inclined cracks on the spatial distribution of magnetic field across the tested specimen. Peak value and amplitude at $200 \mathrm{~Hz}$ are shown to be the best features to construct C-scan images for characterization of crack inclination angles, and the angle discrimination results are evaluated.

\section{Experimental setup}

\subsection{PEC system building}

The built PEC system consists of a data acquisition (DAQ) system (NI USB-6363), excitation circuit, $x-y$ scanner and a PEC probe. The DAQ device is also used to control the motors on the $x-y$ scanner, in addition to picking up the sensed magnetic flux density by SS495A1 Hall sensor placed in the middle of the probe, at a sampling rate of $100 \mathrm{kS} / \mathrm{s}$. Thirty samples of pretriggered signals were also picked up in order to obtain the threshold value of the signal before the excitation. The DAQ device also supplies rectangular waveform of $5 \mathrm{~ms}$ width at $2.5 \mathrm{~Hz}$ to the excitation circuit. The excitation circuit consists of a transistor to amplify the current supplied by DAQ device to $9 \mathrm{~A}$. The number of turns of the excitation coil is 81 while the inner and outer radius are $6.5 \mathrm{~mm}$ and $10.0 \mathrm{~mm}$ respectively. The lift-off is kept constant at 1.0 $\mathrm{mm}$.

\subsection{Details of study}

Five simulated surface cracks of different inclination angles were manufactured in aluminium samples. The crack details are tabulated in Table 1, while Figure 1 provides the illustrations of two of the cracks. 
Table 1. Simulated surface crack parameters

\begin{tabular}{cccc}
\hline $\begin{array}{c}\text { Crack } \\
\text { number }\end{array}$ & $\begin{array}{c}\text { Depth } \\
(\mathrm{mm})\end{array}$ & Width $(\mathrm{mm})$ & $\begin{array}{c}\text { Inclination } \\
\text { angle }\left(^{\circ}\right)\end{array}$ \\
\hline Crack 1 & $8 \pm 0.2$ & $0.35 \pm 0.05$ & $30 \pm 0.5$ \\
Crack 2 & $8 \pm 0.2$ & $0.35 \pm 0.05$ & $45 \pm 0.5$ \\
Crack 3 & $8 \pm 0.2$ & $0.35 \pm 0.05$ & $60 \pm 0.5$ \\
Crack 4 & $8 \pm 0.2$ & $0.35 \pm 0.05$ & $75 \pm 0.5$ \\
Crack 5 & $8 \pm 0.2$ & $0.35 \pm 0.05$ & $90 \pm 0.5$ \\
\hline
\end{tabular}

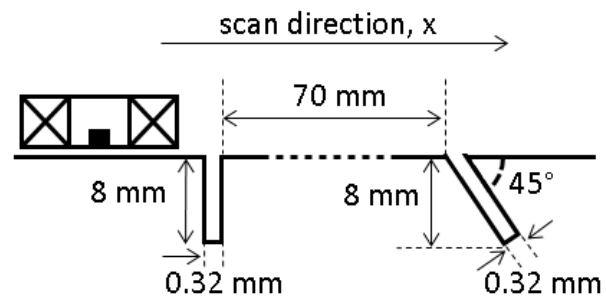

Figure 1. Dimensions of cracks of $90^{\circ}$ and $45^{\circ}$ inclined to the sample surface

The probe is scanned over the aluminium sample to cover an area of $30 \mathrm{~mm} \times 30 \mathrm{~mm}$ at $0.5 \mathrm{~mm}$ intervals, starting at approximately $15 \mathrm{~mm}$ away from the middle of the crack opening to approximately $15 \mathrm{~mm}$ away on the other side of the cracks in the x-direction. At each position, five response signals were picked up and averaged. The scanning lines were perpendicular to the crack line to ease up the analysis. The signals were then normalized. Consequently, differential signals were obtained by subtracting normalized reference signal from the normalized response signals. It is also important to note that the differential signals were obtained at only the rising edge of the response signals. Five features were extracted from the differential signals in both time and frequency domains, namely peak value, peak arrival time, amplitude at fundamental frequency (representing low frequency components), amplitude at 10 $\mathrm{kHz}$ (representing medium frequency components) and amplitude at $50 \mathrm{kHz}$ (representing high frequency components). Spatial positions assignment is explained in the next section.

\section{Results and discussions}

\subsection{Peak shift in the five features using finite element analysis}

Normal surface cracks yield differential signals at the middle of the crack opening stronger than other spatial locations. The most obvious feature to evaluate this effect is the peak value, which reaches the maximum at the middle of the crack opening. This benefits the analysis to effectively locate the location of the surface crack. However, it was empirically observed that cracks with inclination angles other than $90^{\circ}$ tend to produce a different trend. The features tend to reach their maximum or minimum values away from the middle of the crack opening. This observation was verified with finite element modelling (FEM), which was built according to the parameters of the PEC system.

The COMSOL Multiphysics FEM simulation was computed with time-dependent study, where both response and reference signals were obtained for inclined cracks of $60^{\circ}$ and $90^{\circ}$ at $1.0 \mathrm{~mm},-0.5 \mathrm{~mm}, 0 \mathrm{~mm}, 0.5 \mathrm{~mm}$ and $1.0 \mathrm{~mm}$ away from the middle of the crack opening. Noting that $60^{\circ}$ surface crack grows to the right-hand side of the crack opening, negative spatial positions are denoting the positions on the left-hand side of the crack opening. Two time domain features, namely peak value and peak arrival time, were extracted from the differential signals. In the frequency domain, Fast Fourier Transform (FFT) were applied to extract the features from the signals.

Plotting peak value on a graph corresponding to its spatial location shows that the $90^{\circ}$ surface crack has the highest peak value at $0 \mathrm{~mm}$, which is at the middle of the crack opening. Results on $60^{\circ}$ crack, however, gives the highest peak value at $0.5 \mathrm{~mm}$ instead, as shown in Figure 2 (a). This can be explained with the theory of skin depth, where most currents are present at the crack face, which is also treated as a surface. At $0.5 \mathrm{~mm}$ position, on $60^{\circ}$ crack, eddy current is present at the surface of the sample, as well as at the crack face which grows up 
to more than $4.6 \mathrm{~mm}$ away from the opening of the crack. The lowest value of peak arrival time, on the other hand, can't be accurately determined due to the low sampling resolution. The graph in Figure 2 (b) still shows the symmetricity of the $90^{\circ}$ peak arrival time due to the symmetrical effect of the crack. As aforementioned, $60^{\circ}$ grows to the positive positions, consequently causes the trend of the peak arrival time to be skewed to the right.

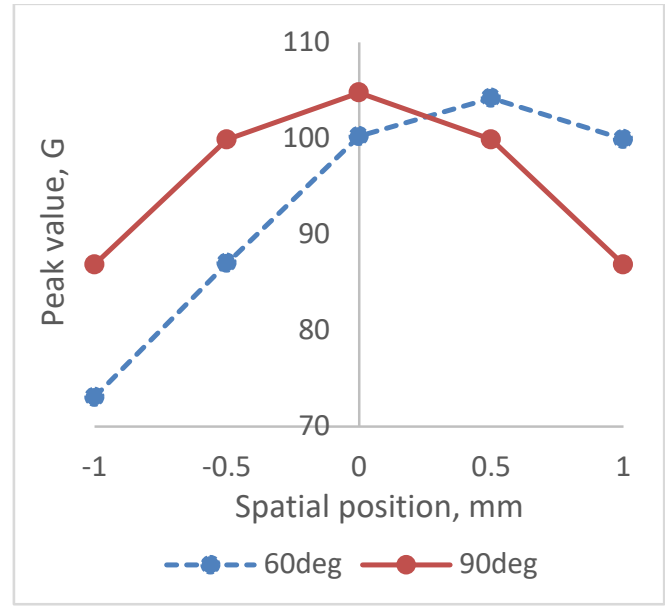

(a)

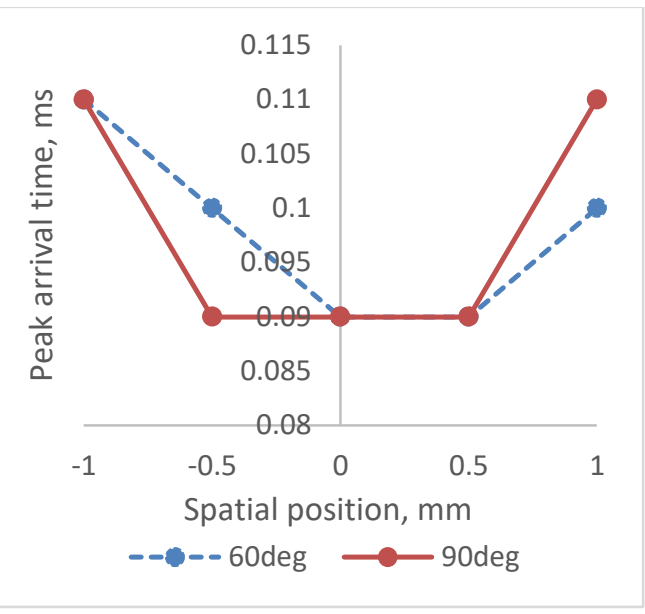

(b)

Figure 2. FEM results on (a) peak value and (b) peak arrival time for inclination of $90^{\circ}$ and $60^{\circ}$

Figures 3(a)-(d) display the analysis in frequency domain. Looking at Figure 3(a) and Figure $3(\mathrm{~b})$ which represents $60^{\circ}$ crack signal, at low frequency, the highest loss of the induced eddy current is at the location of $0.5 \mathrm{~mm}$, followed by $1.0 \mathrm{~mm}, 0 \mathrm{~mm},-0.5 \mathrm{~mm}$ and $-1 \mathrm{~mm}$. This is justified by the presence of crack as seen at each position, where at $0.5 \mathrm{~mm}$ and $1.0 \mathrm{~mm}$, the crack is at the deeper region of the sample. Position of $0.5 \mathrm{~mm}$ has a higher amplitude than 1.0 $\mathrm{mm}$ at low frequency because of the deeper crack location at $1.0 \mathrm{~mm}$, which consequently allows more loss of secondary magnetic flux density before reaching the sample surface. Conversely, at higher frequencies, $0 \mathrm{~mm}$ position shows the highest amplitude as that specific position locates the crack opening.

It can also be seen that $90^{\circ}$ crack exhibits a symmetrical trend across similar spatial distances from the crack opening, where the stem plot for $-1 \mathrm{~mm}$ and $-0.5 \mathrm{~mm}$ are barely seen in Figures 3(c) and (d). As $90^{\circ} \mathrm{crack}$ is perpendicular to the sample surface, the effect of the crack on the spatial location is symmetrical on both positive and negative positions.

This subsection is important to be mentioned to avoid confusion on the spatial positioning in experimental results. Although according to FEM, each feature clearly shows different spatial location of the maximum and minimum values, the spatial positions of the Cscan images constructed from experimental results were assigned based on the maximum peak value obtained across the scanned area. As the scanning was done perpendicularly to the crack, the column which contains the maximum peak value for any row of the image was assigned as the $0 \mathrm{~mm}$ position, while the arrays on the left hand side of the maximum peak value column were assigned as negative positions of interval of $0.5 \mathrm{~mm}$. The arrays on the righthand side of the maximum peak value column were otherwise assigned as positive positions. For all images, despite the feature used, the positions based on the maximum peak value were kept to avoid confusion during analysis. 


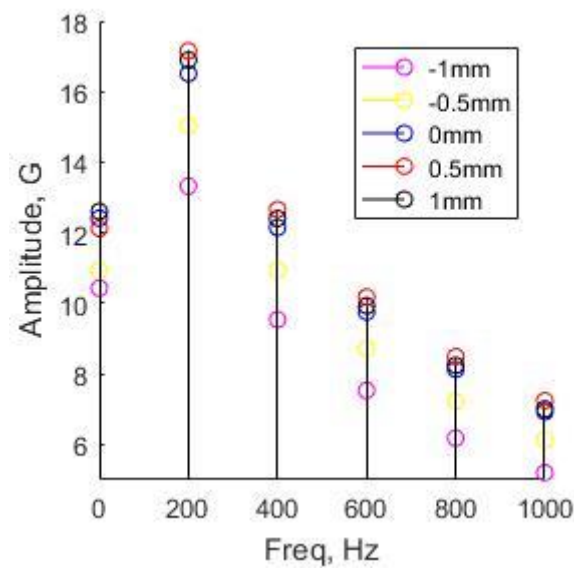

(a)

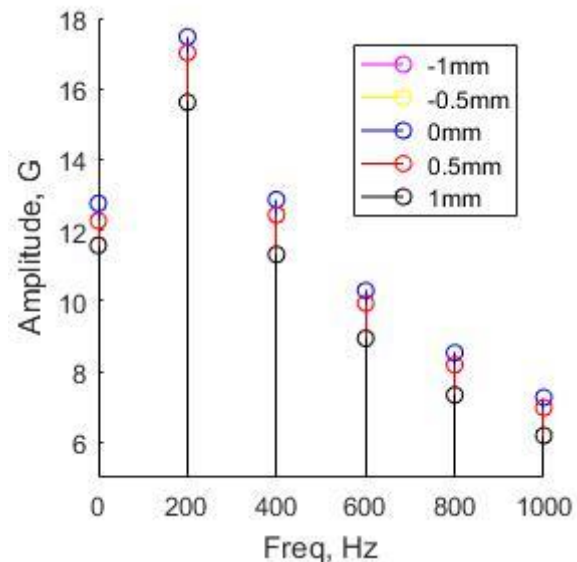

(c)

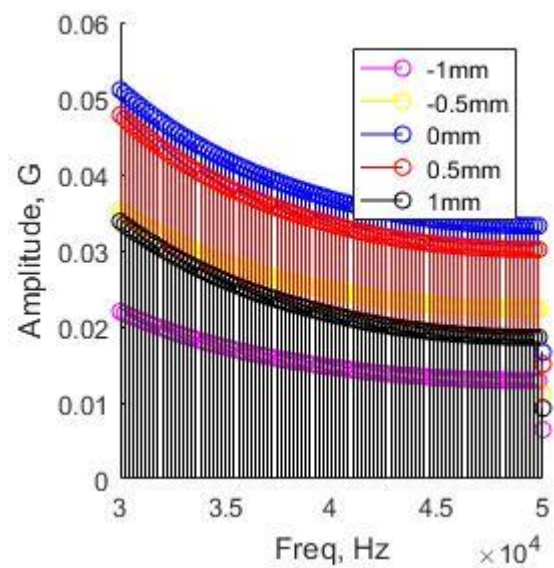

(b)

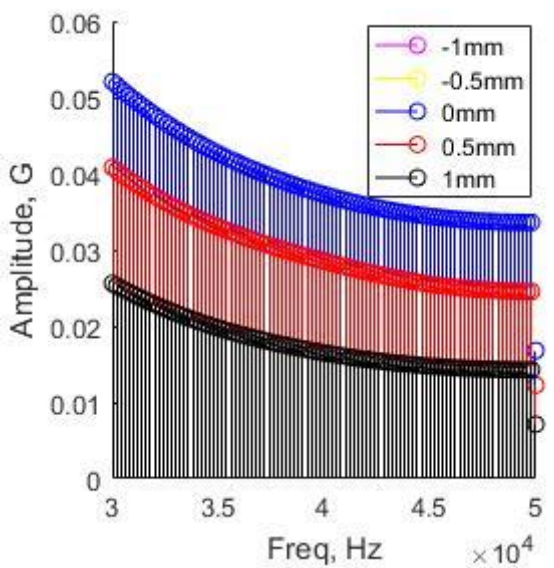

(d)

Figure 3. FFT applied on differential signal from FEM where (a) represents the low frequency components of $60^{\circ}$ crack, (b) high frequency components of $60^{\circ}$ crack, (c) low frequency components of $90^{\circ}$ crack and (d) high frequency components of $90^{\circ}$ crack.

\subsection{Different features for $\mathrm{C}$-scan image construction}

The five features used for $\mathrm{C}$-scan image, peak value, peak arrival time, amplitudes at $200 \mathrm{~Hz}, 10 \mathrm{kHz}$ and $45 \mathrm{kHz}$, were analysed to determine the most intuitive and resourceful features capable of discriminating between cracks of different inclination angles. The images constructed for crack of $30^{\circ}$ inclination and $8 \mathrm{~mm}$ depth using the most discriminative features, peak value and amplitude at $200 \mathrm{~Hz}$, are presented in Figure 4. Referring to Figure 4(a), as the $30^{\circ}$ crack grows from the crack opening on the surface to the positive positions, the distribution of peak values is skewed to the right. Peak values at positive positions settles slower to zero value, at position of approximately $10 \mathrm{~mm}$ as compared to peak values that decreases to zero value at approximately $-6 \mathrm{~mm}$. As peak value represents the degree of severity of the crack, positive positions are more affected by the crack than the negative positions.

As depicted in Figure 4(b), amplitude at $200 \mathrm{~Hz}$ generates a similar trend to peak value. At lower frequencies, the distribution of magnetic field penetrates the specimen is more dispersed, compared to at higher frequencies. The depth of penetration is also higher which permits the information at lower parts of the aluminium sample to be obtained. This allows wider area to be covered by penetration of magnetic field. As penetration of magnetic field of higher frequency components tend to converge from the sample surface to a shallower depth of penetration [14], high frequency components are less affected by the change of the crack structure, which explains a much lower amplitude present at higher frequency components. High frequency components failed to effectively provide C-scan images to explain different 
effects of magnetic field distributions. The images using amplitude at high frequency components are not shown due to the limitation of space. Peak arrival times, on the other hand, contain discrepancies at the left-hand side of the image, due to low signal-to-noise ratios present at low magnitude signals, which makes the region rather noisy. This can be observed better if the scanning area is enlarged to cover more area that is less affected by the crack. However, this is not shown in the paper due to space limitation as well.

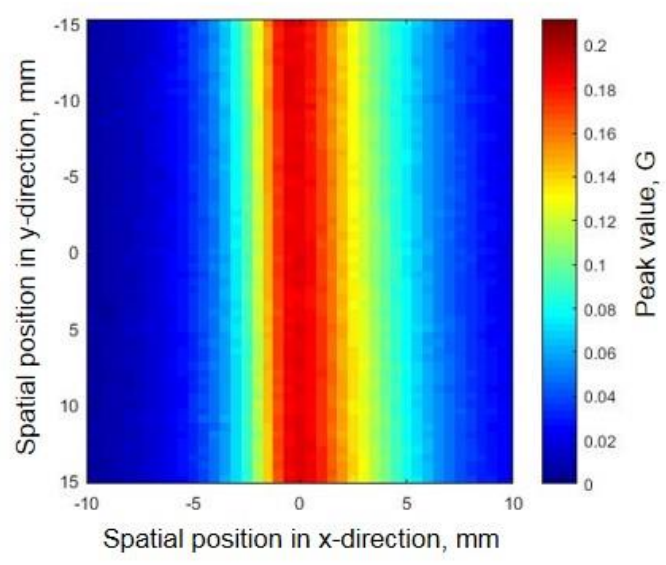

(a)

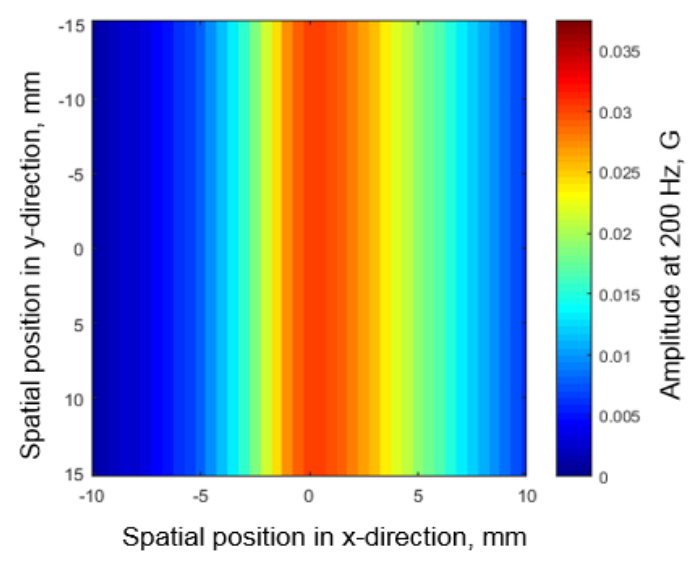

(b)

Figure 4. C-scan images of $30^{\circ}$ crack, using (a) peak value, (b) peak arrival time, (c) amplitude at $200 \mathrm{~Hz}$, (d) amplitude at $10 \mathrm{kHz}$ and (e) amplitude at $45 \mathrm{kHz}$.

\subsection{C-scan images on different inclination angles}

Based on the analysis on different features, peak value was chosen as the feature for analysis of different surface crack inclination angles. Figure 5 (a), (c), (e), (g) shows the remaining four images using peak values representing $45^{\circ}, 60^{\circ}, 75^{\circ}, 90^{\circ}$ crack respectively. Meanwhile, Figure 5 (b), (d), (f), (h) shows the images using amplitude at $200 \mathrm{~Hz}$ representing $45^{\circ}, 60^{\circ}, 75^{\circ}, 90^{\circ}$ crack respectively.

The most obvious trend can be observed when comparing between the images of $30^{\circ}$ and $45^{\circ}$ surface crack with $90^{\circ}$ surface crack. First and foremost, both positive and negative sides of the spatial positions for $90^{\circ} \mathrm{crack}$ are almost symmetrical. Meanwhile the transition gap (yellow, green and turqoise regions) from high peak value to a much lower peak value of $30^{\circ}$ and $45^{\circ}$ crack images are larger on the positive sides, owing to the effect of crack growing to the positive side. The skewness of the image towards the elongation of the inclined crack can be used as a feature to differentiate between inclination angles.

The C-scan images using peak value for $60^{\circ}$ and $75^{\circ}$ do not show much difference when compared to $90^{\circ}$ crack image in terms of the the image symmetricity as the effect of crack elongation is smaller. However, it can still be observed that the transition gap is higher on the positive positions than negative positions. The C-scan images using amplitude at $200 \mathrm{~Hz}$, on the other hand, shows better discrimination ability for $60^{\circ}$ and $75^{\circ}$ cracks. Maximum peak value, although barely visible, is highest for $90^{\circ}$ crack as it has the most effect on top of the crack opening, for both peak value and amplitude at $200 \mathrm{~Hz}$. 
(a)

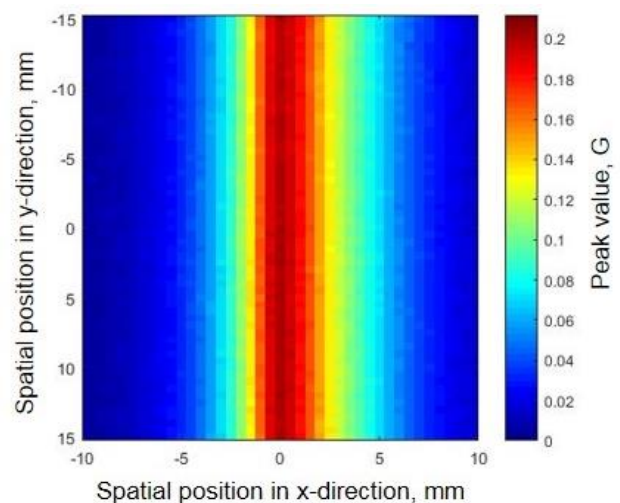

(c)

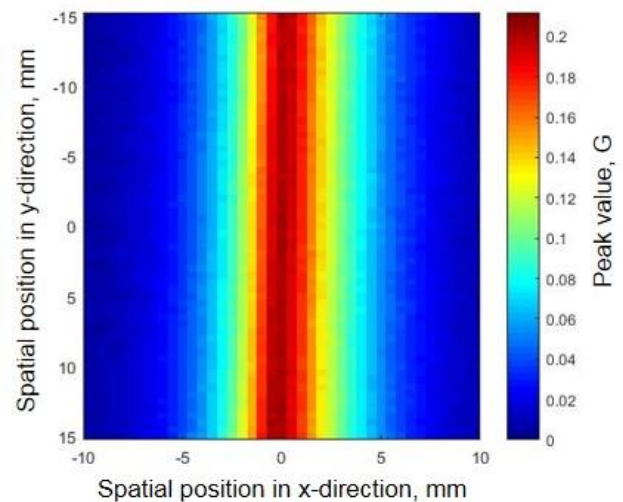

(e)

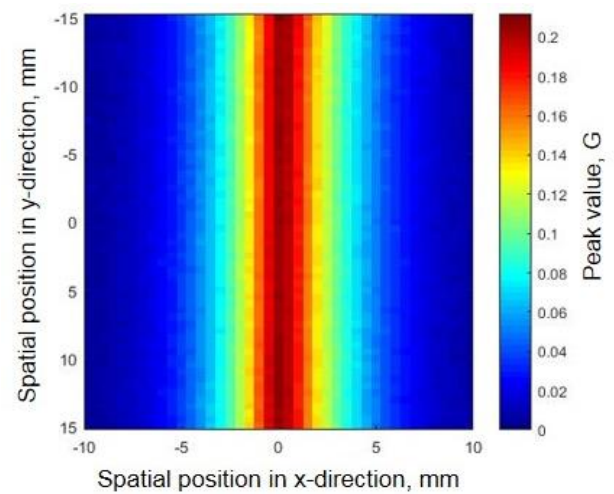

(g)

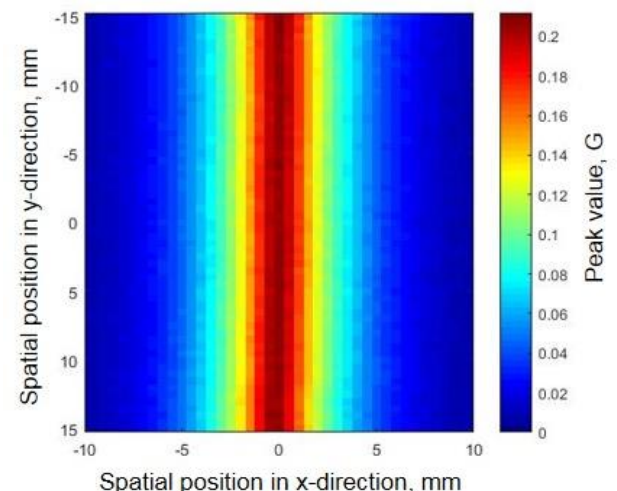

(b)

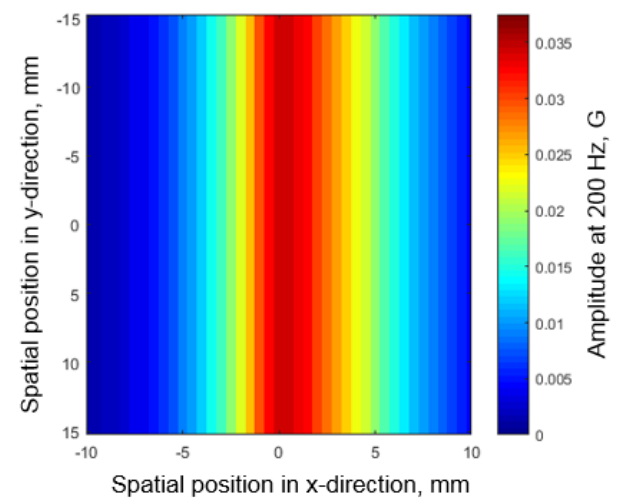

(d)
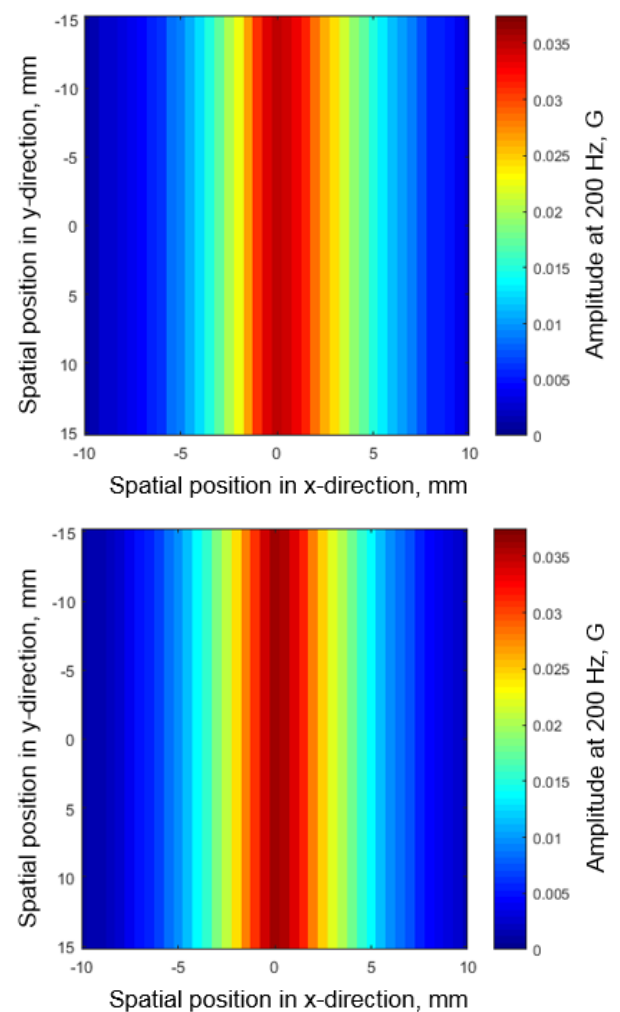

Spatial position in x-direction, $\mathrm{mm}$

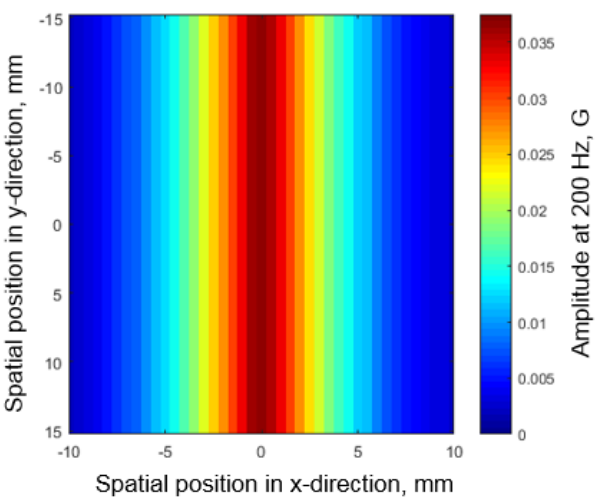

Figure 5. C-scan images of (a), (c), (e), (g) 45ำ $60^{\circ}, 75^{\circ}, 90^{\circ}$ crack respectively using peak value and (b), (d), (f), (h) 45, 60, $75^{\circ}, 90^{\circ}$ crack respectively using amplitude at $200 \mathrm{~Hz}$. 


\section{Conclusion}

FEM analysis on $8 \mathrm{~mm}$ surface cracks of $60^{\circ}$ and $90^{\circ}$ inclination angle provide a preliminary understanding on the effects of inclined cracks on spatial distribution of magnetic field. Features in both time and frequency domains show different trends at different locations according to the severity of the crack effect on the penetration of magnetic field. The location of the maximum peak value for both angles are also different due to the concentration of induced eddy current near the crack face. Experimental results also proved the hypothesis. Additionally, they showed that the two most effective features for inclination angle discrimination to be used were peak value and the amplitude at $200 \mathrm{~Hz}$, with the latter was shown to be on the better side. Both features give asymmetrical trend when constructing C-scan images, except for $90^{\circ}$ cracks. This is driven by the severity of the crack when the crack is grown from the crack opening at the surface to the right-hand side of the crack. From the C-scan images, features can be extracted to further analyse the effect of different angles on the spatial distribution of magnetic field, which will be part of our further work.

\section{References}

[1] $\mathrm{P}$ Gao, C Wang, $\mathrm{Y} \mathrm{Li}$, Z Cong. Electromagnetic and eddy current NDT in weld inspection: A review. Insight - Non-Destructive Test. Cond. Monit. 2015; 57(6).

[2] K N. Azaman, A. Sophian, and F. Nafiah. Effects of Coil Diameter in Thickness Measurement Using Pulsed Eddy Current Non-destructive Testing. IOP Conf. Ser. Mater. Sci. Eng. 2017; 260.

[3] A.Sophian, G Tian, M Fan. Pulsed Eddy Current Non-destructive Testing and Evaluation: A Review. Chinese J. Mech. 2017.

[4] Z. Xu, X. Wu, J. Li, Y. Kang. Assessment of wall thinning in insulated ferromagnetic pipes using the time-to-peak of differential pulsed eddy-current testing signals. NDT E Int. 2012; 51: 24-29.

[5] G Panaitov, HJ Krause, Y Zhang. Pulsed eddy current transient technique with HTS SQUID magnetometer for non-destructive evaluation. IEEE Trans. Appl. Supercond. 2002.

[6] S. Majidnia, R. Nilavalan, Rudlin. A numerical study of the depth of penetration of eddy currents. Insight - Non-Destructive Test. Cond. Monit. 2016; 58(3): 129-134.

[7] Y He, F. Luo, M. Pan. Defect characterisation based on pulsed eddy current imaging technique. Sensors Actuators, A Phys. 2010; 164(1-2): 1-7.

[8] V Arjun, B. Sasi, BPC. Rao, CK. Mukhopadhyay, T. Jayakumar. Optimisation of pulsed eddy current probe for detection of sub-surface defects in stainless steel plates. Sensors Actuators A Phys. 2015; 226: $69-75$.

[9] RF Abrantes, LS Rosado, M Piedade, PM. Ramos. Pulsed eddy currents testing using a planar matrix probe. Meas. J. Int. Meas. Confed. 2016; 77: 351-361.

[10] Y Li, H Jing, I Mukriz, Z Abidin, B Yan, D Li, Y Li, D Zhou. Gradient-field pulsed eddy current probes for imaging of hidden corrosion in conductive structures. Sensors Actuators A Phys. 2016; 238: 251265.

[11] X. Chen, D. Hou, L. Zhao, P. Huang, and G. Zhang. Study on defect classification in multi-layer structures based on Fisher linear discriminate analysis by using pulsed eddy current technique. NDT E Int. 2014; 67: 46-54.

[12] I. Zainal Abidin, G. Yun Tian, J. Wilson, S. Yang, D. Almond. Quantitative evaluation of angular defects by pulsed eddy current thermography. NDTE Int. 2010;43(7): 537-546.

[13] M H. Rosli, R. S. Edwards, Y. Fan. n-plane and out-of-plane measurements of Rayleigh waves using EMATs for characterising surface cracks. NDT E Int. 2012; 49: 1-9.

[14] J García-Martín, J. Gómez-Gil, E. Vázquez-Sánchez. Non-destructive techniques based on eddy current testing. Sensors. 2011; 11(3): 2525-2565.

IJEEI Vol. 5, No. 4, December 2017: $309-316$ 